\title{
Epoxidharz-Allergie: Auch an Härter und Verdünner denken
}

— Das Einsatzgebiet von Epoxidharz hat sich deutlich ausgeweitet. Früher vor allem in der Schwerindustrie eingesetzt, findet es heute bei der Betonsanierung, in Parkhäusern, als Fußbodenbeschichtung oder zu dekorativen Zwecken Verwendung, erläuterte Prof. Dr. Johannes Geier, Göttingen. Bei der Verarbeitung werden zwei Komponenten verwendet, die Harzkomponente, die meist auch noch einen Reaktivverdünner zur Einstellung der Konsistenz enthält, und der Härter. Geschlossene Mischsysteme sind auf dem Markt erhältlich, auf dem Bau wird die Mischung aber häufig noch in offenen Behältern hergestellt, die Kontamination der Kleidung und ein Hautkontakt mit den Chemikalien ist verbunden mit einem deutlich erhöhten Sensibilisierungsrisiko.

Das stärkste Allergen im Epoxidharz ist Bisphenol-A-Diglycidylether (DGEBA, Monomere oder Oligomere), häufig begleitet von einem Epoxidharz auf Basis von Bisphenol-F (DGEBF) mit drei Isomeren. DGEBA wird in der Standardreihe getestet. Weil DGEBA kreuzreaktiv ist mit DGEBF, andererseits auch eine Koexposition mit beiden Harztypen zu einer Kosensibilisierung führen kann, sieht man meist eine gekoppelte Sensibilisierung. Deshalb muss DGEBF nicht regelmäßig neben DGEBA getestet werden, erläuterte Geier.

Ein von der deutschen gesetzlichen Unfallversicherung gefördertes Forschungsprojekt untersucht, welche Allergene in Epoxidharzen maßgeblich sind, um den Herstellern die Möglichkeit zu geben, weniger sensibilisierende Inhaltsstoffe zu verwenden als bisher. Die Daten des Informationsverbund Dermatologischer Kliniken (IVDK) von 41.704 Patienten aus den Jahren 2012 bis 2015 ergaben eine Quote von 1,74\% positiver Reaktionen auf Epoxidharz nach der Standardreihe. Nur ein Drittel der 743 auf DGEBA reagierende Patienten wurde auch mit Reaktivverdünnern oder Härtern getestet. „Das ist viel zu wenig, ich kann nur dringend dazu auffordern, bei positiver DGEBA-Reaktion auf die ganze Epoxidharzreihe zu testen, weil man sonst relevante Sensibilisierungen übersieht."

Bei Test aus Reaktionsverdünner reagierten $48,7 \%$ der nach den IVDK-Daten untersuchten Patienten auch auf 1,2-Hexandioldiglycidylether (1,6-HDDGE). 35,3\% reagierten auf 1,2-Butandioldiglycidylether (1,2-BDDGE), fast immer zusammen mit einer Reaktion auf 1,6-HDDGE. „Die Patienten sensibilisieren sich fast immer primär auf 1,6-HDDGE", ergänzte Geier. Auch auf andere Reaktionsverdünner wie Phenylglycidylether (PGE, 30,3\%), p-tert-Butylphenylglycidylether (PTBRGE) und Cresylglycidether (CGE, 15,7\%) reagierten die Patienten relativ häufig, selten auch auf p-tert-Butylphenylglycidylether (PTBPGE, $2,7 \%)$.

Die häufigsten positiven Reaktionen auf Härter fanden sich bei m-Xylidendiamin (synonym M-Xylendiamin oder M-Xylylendiamin, MXDA, 20,4\%). Die Testsubstanz (MXDA 0,1\% in Vaseline) ist in Deutschland nicht mehr erhältlich, kann aber bei Chemotechnik in Schweden bestellt werden. „Das ist legal und kein Problem, man muss nur dran denken", betonte Geier. „Man übersieht sonst das häufigste Allergen im Härter." Der Härter hat einen niedrigen Dampfdruck und verdunstet als erstes, wenn ein
Fußboden beschichtet wird. Das kann rasch zu einem aerogenen Kontaktexzem führen. Obwohl Isophorondiamin (IPDA) viel häufiger als MXDA eingesetzt wird, wurden Patienten darauf weniger häufig positiv getestet $(7,7 \%)$. Weitere positive Reaktionen wurden auf die Härter Trimethylhexan-1,6diamin (TMHDA, 6,2\%) und Diethylentriamin (DETA, 2,6\%) beobachtet.

Reaktionen auf Reaktionsverdünner und Härter treten nicht nur bei positiv auf DGEBA-Harz getesteten Personen auf. Etwa jeder fünfte auf Reaktionsverdünner reagierende Patient hatte in der Standardreihe nicht auf DGEBA reagiert. Bei Härtern ist dieses Phänomen noch häufiger zu beobachten: 78\% der Patienten mit Reaktionen auf DETA waren negativ auf DGEBA-Harz getestet, bei TMHDA waren es $57 \%$, bei IPDA $33 \%$ und bei MXDA 24\%. „Wenn ein Patient bei Epoxidharzexposition auf DGEBA nicht reagiert, können die Symptome irritativ sein, müssen es aber nicht", betonte Geier. „Im Verdachtsfall sollte daher neben der Standardreihe immer die Epoxidreihe getestet werden.

Ein in Deutschland nicht getesteter und deshalb als wichtiges Allergen übersehener Härter ist 2,4,6-Tris (dimethylaminomethyl)phenol (tris-DMP), nach einer finnischen Studie der zweithäufigste Härter in Epoxidharzen. „Tris-DMP wird auch in Deutschland viel verwendet, aber wir entdecken das nicht mangels Testsubstanz", gab Geier zu bedenken. „Erfreulicherweise gibt es seit Anfang des Jahres tris-DMP als Testsubstanz in einer Konzentration von $0,5 \%$ in Vaseline von Chemotechnik in Schweden. Ich kann nur dringend dazu raten, das zu bestellen und zu berücksichtigen."

Friederike Klein

AllergoCompact: „Allergie und Beruf“

\section{Provokationstest bei Arzneimittelallergie: Goldstandard oder nicht?}

— Waren anfangs bei der Abstimmung im Saal noch $72 \%$ der Anwesenden der Meinung, bei einer Arzneimittelallergie sei der Provokationstest der Goldstandard, befanden sich die Befürworter der These nach dem Streitgespräch mit $48 \%$ in der Minderheit. Um es vorwegzunehmen: Es war eine Frage der Begrifflichkeit, um die die beiden Experten Prof. Dr. Axel Trautmann, Würzburg, und Prof. Dr. Randolf Brehler, Münster, rangen. Und so änderte auch ein großer Teil der Anwesenden im Saal im Laufe der Pro- und Kontra-Diskussion seine Meinung.
Wenn nach der Einnahme von Medikamenten objektive und subjektive Beschwerden wie Urtikaria, Exanthem, Schwindel, Schweißausbrüche, Benommenheit, Brustschmerzen, Zittern oder Übelkeit auftreten, werden in der Regel Prick- und Intrakutantests und eine IgE-Diagnostik durchgeführt. Doch nur für wenige Medikamente, so Trautmann, seien die Tests sensitiv genug, um damit verlässliche Aussagen treffen zu können. Nur ein positives Ergebnis im Provokations- 